\title{
USED EQUIPMENT FOR SALE
}

- E. Fjeld, Co. reconditions a variety of scanning electron microscopes (starting at $\$ 25,000$ ) to meet the needs of the secondary market. Energy dispersive X-ray systems are also available. Contact E. Fjeld Co., 3 Executive Park Drive, North Billerica, MA 01862, (508)667-1416.

- Hitachi S-520 SEM with image manipulation and alphanumeric generator. Includes pump, water recirculator, manuals and spare parts kit. Asking $\$ 25,000$. Contact Gloria Hopkins, Tegal Corp., Tel: (707) 763-5600 X5316.
- Hitachi H-600 TEM and Kevex Quantex VI EDXA combined:

TEM includes $100 \mathrm{KV}$ accelerating voltage and $300 \mathrm{KX}$ mag, 2A lattice resolution and $4.5 \mathrm{~A}$ point/point resolution. Also double-tilt \& rotation holders, SAED, HREM, UHD and analytical modes. STEM unit available.

EDXA includes diffraction analysis and both qualitative and quantitative software. Also Winchester with dual Iomega drives.

Contact Mike Novak, The Earth Technology, Tel: 609)482-5553.
- JEOL JEM-100CX TEMSCAN with side entry goniometer (SEG) and high resolution scanning attachment (ASID). Under continuous service contract and is in good working condition. Includes wave form monitor, Liquid nitrogen baffle, Penning guage, image selector switch (IMS) and multiple display device (MDD). $\$ 50,000$ or best offer. Contact: Dr. Elizabeth D. Hay, Harvard Medical School, (617) 432-1651.

\section{STUATIONS WANTED}

Individuals seeking employment who are currently employed may run a "blind" advertisement, in which case a Microscopy Today box number will be used for responses. The responses, in turn will be promptly forwarded to the advertiser. Any response from the advertiser's current employer will not be forwarded.

- Seeking an EM management position in an active biological research laboratory. Over six years of active experience in the management and operation of multidisciplinary cell biology research laboratories. Knowledgeable in SEM, TEM, LM and cell culture techniques. Trained students (including postdocs) in electron and light microscopy. BA in Biology/Biological Science. Contact for complete resume. Bobbie Lee Schneider, (619) $792-2454$.
- Recent MS (Michigan State) in Metallurgical Engineering. Two plus years previous experience as QA Lab Manager (cold mill) for National Steel. Experience with SEMs, TEMs, EDS, WDA and X-ray diffraction. Seeking position in research or product development. James Stout: (517) 336-7430.
- Recent graduate with BS in biology and formal EM training, plus experience with SEMs, TEMs and EDS, is seeking challenging position. Location open. Paula Hnatew: (717) 347-7989. 